\title{
Trivium
}

Revue franco-allemande de sciences humaines et sociales - Deutsch-französische Zeitschrift für Geistesund Sozialwissenschaften

$9 \mid 2011$

Vitesse et existence. La multiplicité des temps historiques

\section{Von der Universalgeschichte zur Globalgeschichte? Zeiterfahrungen}

\author{
François Hartog \\ Traducteur : Bernd Schwibs
}

\section{OpenEdition}

Journals

Édition électronique

URL : http://journals.openedition.org/trivium/4059

DOI : $10.4000 /$ trivium.4059

ISSN : 1963-1820

Éditeur

Les éditions de la Maison des sciences de l'Homme

\section{Référence électronique}

François Hartog, « Von der Universalgeschichte zur Globalgeschichte? Zeiterfahrungen », Trivium [Online], 9 | 2011, online erschienen am 30 November 2011, abgerufen am 21 Dezember 2020. URL : http://journals.openedition.org/trivium/4059; DOI : https://doi.org/10.4000/trivium.4059

Ce document a été généré automatiquement le 21 décembre 2020.

Les contenus des la revue Trivium sont mis à disposition selon les termes de la Licence Creative Commons Attribution - Pas d'Utilisation Commerciale - Pas de Modification 4.0 International. 


\title{
Von der Universalgeschichte zur Globalgeschichte? Zeiterfahrungen
}

\author{
François Hartog \\ Traduction : Bernd Schwibs
}

\section{NOTE DE L'ÉDITEUR}

Wir danken François Hartog und der Zeitschrift Le Débat für die freundliche Genehmigung, diesen Artikel zu übersetzen.

1 In Europa hat der Sinn der Geschichte - ob darunter nun Bedeutung, Zweck oder einfach ihre Richtung verstanden wird - den Prüfungen und Verbrechen des 20. Jahrhunderts nicht standhalten können; und mit ihm ist auch der Begriff der Universalgeschichte, den das 19. Jahrhundert so hochgehalten hatte, zutiefst beschädigt worden. Sinn der Geschichte, aber nicht minder auch Sinn des Menschen oder Sinn der Kultur. Nennen wir nur Heidegger, Freud oder Valéry. Der Krieg von 1914 mit seinen Millionen von Toten, seinen Trümmern und den ihm entspringenden radikalen Umwälzungen zeitigte nicht nur eine dauerhafte und vielförmige Infragestellung dieses Begriffs, sondern auch brutale, jeglichem Selbstzweifel enthobene und tödliche Reaffirmierungen. Die Folgen des Zweiten Weltkriegs oder, genauer, seine Spätwirkungen, haben das Fragen nach der Sinnlosigkeit ${ }^{1}$ der Geschichte - deren fehlenden Sinn oder Sinnverlust - bis zum Äußersten getrieben. ${ }^{2}$

2 Doch seit dem Fall der Berliner Mauer und dem Vordringen der so genannten Globalisierung wurde hier und dort das Thema wieder spruchreif, jedenfalls das des Sinns der Geschichte, während zugleich die Ideologie, die dessen universelle Geburtshelferin sein wollte, ihre Verheißungskraft verloren hatte. ${ }^{3}$ Für mich geht es hier und jetzt natürlich nicht darum, selbstherrlich zu verfügen, dass es einen Sinn der Geschichte gebe oder auch nicht, oder dass es erneut einen Sinn der Geschichte geben könnte (und wäre es auch der ihres proklamierten »Endes«), sondern nur darum, als Historiker besser zu begreifen, was sich unlängst ereignet hat, sich gerade ereignet. 
Jenseits der Lobeshymnen auf die Globalisierung oder deren Anprangerung gab es durchaus Beobachter, die das gleichsam Hand in Hand gehende Fortschreiten des Globalen und des Lokalen, der Uniformisierung und der Differenzierung festgestellt haben. Je mehr das Globale den Sieg davonzutragen scheint, je tiefer es in Lebensrhythmen und Lebensweisen eingreift, umso nachhaltiger sucht sich das Bemühen um Unterschiede geltend zu machen und Anerkennung zu gewinnen. Was im Besonderen in den 1980er Jahren vielerorts zum Einsatz und zur Mobilisierung jener dann herrschenden Begriffe geführt hat: Erinnerung (mit der Pflicht zur Erinnerung), Identität (mit ihrem Streben und Verlangen nach Anerkennung) und Erbe (zumal in der Form des von der UNESCO geförderten »Welterbes«). Angesichts dieser Phänomene sind mehrere Diskurse zu vernehmen: An dem einen Pol haben wir den Diskurs der Tenöre der `Deterritorialisierung`, der `Zirkulation` und der `Ströme`, am anderen Pol macht sich die Forderung nach vermehrter lokaler oder gruppenspezifischer Erinnerung und Identität hörbar - und zwischen beiden, sich von beiden absetzend, erheben jene ihre Stimme, die kommunitaristische Rückzüge befürchten und das Zusammenlebens der nationalen Einheiten in Gefahr sehen.

4 Die hier vorgeschlagene Reflexion über die Zeiten der Welt erhebt nicht den Anspruch, auf diese allzu massiven Fragen eine Antwort zu geben; sie schlägt eine Klärung vor. Von Erfahrungen der europäischen Welt aus möchte sie sich einer vergleichenden Perspektive oder einem vergleichenden Horizont einschreiben. Es geht, genauer gesagt, darum, einige Zugänge vorzuschlagen, mit denen ein Vergleich verschiedener europäischer wie nicht-europäischer - Zeiterfahrungen ermöglicht wird. Programmatisch werde ich zwei erste mögliche Pisten angeben, die sich in zwei anfangs unterschiedlichen, aber potentiell konvergenten Registern entfalten.

Die erste Piste ist die des >Historizitätsregimes` [régime d'historicité], eines Arbeitsund weiter zu bearbeitenden Begriffs, den ich erstmals 2003 in meinem Buch Régimes $d^{\prime}$ 'historicité ${ }^{4}$ entfaltet habe. Eine Reflexion über die Art Universalgeschichte, wie sie Europa hervorgebracht und ab dem 19. Jahrhundert angewandt hat, liefert einen zweiten Zugang: ihr Erfolg, ihre unzweideutige Evidenz, der Misskredit, in den sie kaum ein Jahrhundert später geriet, ihr jüngstes und nicht unumstrittenes Wiederaufleben. Zusammen mit einer präziseren Frage: Welche Temporalität setzte diese Universalgeschichte in ihren Anfängen voraus? Wie kam es dazu, dass sie in den 1920er Jahren von Dekadenz- und Endzeitstimmungen heimgesucht wurde? Kann es für einen solchen Begriff heute noch einen Platz geben, und wenn ja, wie wäre er $\mathrm{zu}$ formulieren?

\section{Das ıHistorizitätsregime` als Vergleichsinstrument}

6 Unter >Historizitätsregime` verstehe ich eine je spezifische Verknüpfung der Kategorien Vergangenheit, Gegenwart und Zukunft. Die Zeitordnung ist nämlich eine andere, je nachdem, ob die zentrale Betonung auf der Vergangenheit, der Zukunft oder der Gegenwart liegt. Das `Historizitätsregime` ist keine fix und fertige Realität, sondern ein heuristisches Instrument. Ohne irgendeine direkte denotative Funktion, führt es uns in die Nachbarschaft des Weberschen Idealtypus. Zwar hilft es, die okzidentalen Erfahrungen von Zeit verstehbarer zu machen, ist aber - und darum genau geht es strukturell weder eurozentriert noch eurozentristisch. Ausgehend von Überlegungen Claude Lévi-Strauss' zu Ethnologie und Geschichte und unter Rückgriff auf Arbeiten des 
amerikanischen Ethnologen Marshall Sahlins über die Gesellschaften der Maori habe ich mich in dem erwähnten Buch bemüht, den Begriff darzulegen und ihn nach Möglichkeit plausibel zu machen. ${ }^{5}$

Um es auf eine allzu schematische (und damit womöglich mechanistisch wirkende) Weise $\mathrm{zu}$ sagen: Die Erfahrung Europas lässt sich unter drei zentralen `Historizitätsregimen` fassen, nämlich: ‘altes Regime`, `neuzeitliches Regime` und >christliches Regime`, welch letzteres weder mit den beiden anderen verschmilzt noch vollkommen von ihnen getrennt ist. Da es Gegenstand gesonderter Überlegungen ist ${ }^{6}$, sei es hier ausgeblendet. Wer weiß, vielleicht wohnen wir heute dem Auftreten eines neuen Regimes bei, in dem, parallel zur Globalisierung, die Kategorie der Gegenwart dominant wird? Das ist zumindest die Hypothese, die ich auf die Probe stellen möchte, und zwar mit dem Hauptziel, unsere Gegenwart besser zu erfassen.

Das ıalte Historizitätsregimer entspricht dem großen Modell der historia magistra vitae: Es beruht auf Parallelisierung, beruft sich auf Lehren der Geschichte und setzt auf Nachahmung. Die beispielhafte Vergangenheit ist nicht (wirklich) vergangen, weil nicht überholt. Gibt es ein Goldenes Zeitalter, so liegt es hinter uns. Die Zeit schreitet nicht voran. In Griechenland schon im 4.Jahrhundert vor unserer Zeitrechnung konzipiert, ist dieses Modell zwar gelegentlich in Frage gestellt worden, aber bis ins 18. Jahrhundert wirksam geblieben.

Doch ab Ende des 18. Jahrhunderts macht Europa die Erfahrung einer Verzeitlichung der Geschichte: Zur Idee des Fortschritts hinzu kommt die einer Geschichte - einer Geschichte schlechthin / kat'exochen -, die als Prozess und sogar mehr noch: als Seinerselbst-Innewerden in der Zeit verstanden wird. In mittlerweile klassischen Texten hat Reinhard Koselleck scharfsinnig und subtil die Herausbildung dieses modernen Geschichtsbegriffs erhellen können, dabei zugleich die Richtung gewiesen für eine historische Semantik, mit der ebenfalls sein Name verbunden ist. ${ }^{7}$ Seither ist Zeit nicht mehr nur der Rahmen dessen, was geschieht, die Dinge ereignen sich nicht mehr in der Zeit, sondern durch sie: Zeit wird zum Akteur. Die geschichtliche Zeit erwächst genau aus der Spannung zwischen Erfahrung und Erwartung. ${ }^{8}$ Sodass 1789 als (zumindest symbolisches) Datum für den Übergang des ralten in das ıneue Historizitätsregimer gelten kann. Im ralten Regimer wandte man sich der Vergangenheit $\mathrm{zu}, \mathrm{um} \mathrm{zu}$ begreifen, was sich ereignete, war es doch ausgemacht, dass Verstehen von der Vergangenheit hin zur Gegenwart und zur Zukunft verläuft. Im sneuen Regimes dagegen wird die Kategorie der Zukunft vorrangig: Aus der Zukunft leuchtet fortan das Licht, das die Gegenwart wie auch die Vergangenheit verständlich macht. Ihr muss folglich entgegengegangen werden. Die Zeit wird - häufig schmerzhaft - empfunden als Beschleunigung; an die Stelle des (einstigen) Beispielhaften ist das Einzigartige getreten. Das Ereignis wird das, was sich nicht wiederholt. Damit ist der Einstieg in ein > futuristisches Regimer erfolgt.

\section{Die Historie der Historiker}

Das gesamte europäische 19. Jahrhundert über, während sie sich institutionalisierte und den ehrgeizigen Versuch unternahm, sich - nach dem Modell der Naturwissenschaft - als Wissenschaft zu präsentieren, hat die Geschichtswissenschaft sich auf eine historische Zeit gestützt und sie in Szene gesetzt; eine lineare, kumulative und irreversible Zeit, die einer politischen Geschichtsschreibung entsprach, in der 
Nationen die Fürsten als geschichtliche Akteure ablösten und Fortschritt das Heil ersetzte. Diese neue Geschichtswissenschaft war genau jene, die das folgende Jahrhundert dann, unter Aufgabe des Nationalen zugunsten des Sozialen, als "historisierende«, "Ereignisgeschichte» oder »erzählende Geschichte« abwertete. Allerdings bedurfte es dafür der vorgängigen vielfältigen Kritik und Infragestellung seitens vor allem der Philosophie wie auch jener Neuankömmlinge: Ökonomie, Soziologie und Psychologie. Karl Marx entwirft seine Fundamentalkritik des Kapitalismus und bindet sie ein in die machtvolle Maschinerie der Klassenkämpfe, während Henri Bergson das Bewusstsein der Dauer einführt, von dem Proust für die Literatur und Péguy für die Geschichte meisterhaft Gebrauch machen werden. In Deutschland stehen sich Ende des Ersten Weltkriegs mit der "Krise des Historismus" die Verfechter einer wissenschaftlichen Geschichte (die Neukantianer) und jene gegenüber, die den Vorrang der gelebten Erfahrung, des Erlebnisses, verteidigen (Dilthey). Andererseits lenken die Wirtschaftskrisen des 19. Jahrhunderts ihre Beobachter hin zum Begriff der Zyklen, der zugleich dazu zwingt, sich von der ausschließlich linearen Zeit oder von einer schlicht linearen, kumulativen und fortschreitenden Zeit zu verabschieden. Mag der Lauf des Fortschritts auch unbegrenzt sein, so hat er doch nicht minder seine Phasen des Aussetzens, Vorpreschens und Nachhinkens.

11 In Frankreich werden die Historiker zu jener Zeit - nicht zuletzt von François Simiand, dem talentierten Fürsprecher der noch jungen Durkheimschen Soziologie - aufgerufen, sich vom Akzidentellen abzuwenden und sich stattdessen mit dem Regelmäßigen und Repetitiven zu beschäftigen..$^{9}$ Einige stürzen sich auf die Geschichte der Preise, so Ernest Labrousse, der 1932 seinen Esquisse du mouvement des prix et des revenues en France au XVIII siècle veröffentlicht. Doch zum eigentlichen Gegenstand einer bald schon als »Dialektik der Dauer « bestimmten Geschichte wird »die Pluralität der sozialen Zeit» Anfang der 50er Jahre bei Fernand Braudel. Dessen erster und rasch berühmt gewordener Versuch war Das Mittelmeer und die mediterrane Welt in der Epoche Philipps II., der auf drei Stufen der Zeitlichkeit aufbaut: ${ }^{10}$ zunächst dem Sockel der longue durée, der »langen Dauer«, dann der Konjunktur, schließlich der kurzen Zeitdauer des Ereignisses. Von den drei dramatis personae war die letzte, die der kurzen Zeitdauer, die bekannteste und zugleich auch die am wenigsten interessante: Es ist die der klassischen politischen Geschichte. Die zweite, die der Zyklen und Zwischenzyklen, war gerade dabei, sich einen anerkannten Platz zu erobern. Mit ihr kann der Historiker eine Erzählung konstruieren, die Braudel das »Konjunktur-Rezitatif» nennt. Die noch unbekannte dritte Figur stellte den neuartigsten Beitrag dar. Sie forderte auf, die Geschichte und ihre Rhythmen von jenen "Schichten langsam verlaufender Geschichte« aus, die »an der Grenze von Bewegung überhaupt « stehen, neu zu überdenken. Hier sollte der Historiker auf die Ebene mit der höchsten Erklärungskraft stoßen, lassen sich doch in dieser Tiefe jene "Strukturen" ausmachen, die ihm als privilegierter Gegenstand gelten. Und dorthin wird Braudel auch ein letztes Mal zurückkehren, wenn er sich auf die Suche nach der Identität Frankreichs macht, dem Thema seines letzten Buches. ${ }^{11}$

Dieses Temporalitätsmodell hat sich als fruchtbar erwiesen. Es hat in den 1960er Jahren den Blick vieler Historiker in Frankreich und anderswo verändert und deren Fragenkatalog bereichert. Am Rande sei dennoch festgehalten, dass es die Frage des Ineinandergreifens bzw. der Verknüpfung der drei Temporalitäten - die weiterhin an einer ihnen äußerlich bleibenden Zeit gemessen werden - noch offen lässt. Es sei denn, 
gerade die Sprünge zwischen den Stufen bzw. Schichten wären aufschlussreich, soll durch sie doch Unerwartetes und Neues zutage treten können. Eine Gesellschaft, darauf darf man wetten, hat einer zutreffenden Bemerkung Jacques Revels zufolge nie vollkommen die Konjunktur ihrer Strukturen und noch weniger alle Ereignisse ihrer Konjunktur. Treiben wir Braudels Hypothese noch etwas weiter, dann löst die Zeit der Geschichte sich auf in eine Vielzahl partieller, lokaler oder spezifischer Zeiten, da nicht nur jeder einzelne Prozess, sondern auch jede einzelne Praktik, die der Historiker sich zum Gegenstand erwählt, mit einer eigenen Zeitlichkeit versehen oder, besser noch, davon nicht zu trennen ist. Damit gilt: Keine feingesponnene Sozialgeschichte ohne Berücksichtigung dieser vielfältigen Temporalitäten und der durch ihre Unterschiede induzierten Effekte: Zeitgenössisches und Unzeitgenössisches gibt es auf jeder Stufe, in jedem Winkel, sogar in jedem ihrer Bewohner, oder auch: Gleichzeitigkeit von beidem. Jedenfalls wird Geschichte zum Feld vielfältiger, wenn nicht gar auseinanderfallender Temporalitäten, bleibt aber dennoch, dies sei wiederholt, ein äußerer Maßstab (kalendarische, astronomische Zeit), der jede einzelne Temporalität misst, bleibt jene »exogene«, »den Menschen äußerliche« Zeit: »die gebieterische Zeit der Welt«, um noch einmal Braudel zu zitieren. ${ }^{12}$

Und noch eine Frage lässt das Braudelsche Modell unbeantwortet, nämlich: Welchen Stellenwert nehmen darin die Zeitvorstellungen der anderen ein, die nicht-westlichen Temporalitätsformen? Mit dieser Frage erhebt sich eine weitere: Wo ist das Braudelsche Modell der Temporalitäten im Verhältnis zum sneuzeitlichen Historizitätsregime` einzuordnen? Handelt es sich dabei nicht um eine - aus dem Inneren der Disziplin selbst heraus erfolgende - Ablehnung der zukunftsorientierten Haltung? Die Distanzierung vom Ereignis, die Stufeneinteilung der Zeiten, das Gewicht, wenn nicht die Last der longue durée, der »langen Dauer«: all das sind ja Äußerungen dieser Ablehnung. Die Menschen machen die Geschichte, aber sie wissen nicht, dass sie sie machen, nimmt Braudel seinerseits Marx' Diktum wieder auf, das heißt, sie machen sie unter Bedingungen, die ihnen weitgehend entzogen sind. Zwar ist die Zeit ein gebieterischer Akteur, ja der Hauptakteur, doch er entzieht sich dem Zugriff. Es gibt die Last der »langen Dauer « oder auch die extreme Trägheit, Langsamkeit der Struktur, und sie könnte nahezu als eine verzeitlichte Form des Schicksals erscheinen. Findet sich darin etwas von Marx wieder, dann ohne die Utopie oder den futuristischen Glanz der Revolution. Auch etwas von Fustel de Coulanges steckt darin, für den weit eher die Institutionen die Menschen machen als dass sie von diesen gemacht werden. ${ }^{13}$ Tatsächlich wird die longue durée beschrieben als »Wasseroberfläche«, die alles mit sich fortträgt. ${ }^{14}$

\section{Philosophien der Geschichte und Universalgeschichten}

Greifen wir zeitlich zurück und kommen wir noch einmal, freilich auf eine andere Weise, für einen Augenblick auf das ıneuzeitliche Historizitätsregime` zu sprechen. Entdeckung und Gestaltung der vom Fortschritt regierten Prozessgeschichte gingen einher mit der sich ihrer selbst und ihres Sieges sicheren, glücklichen Zeit der Geschichtsphilosophien, der Universal- oder Kulturgeschichten. Wie schon François Guizot in seiner Vorlesung an der Sorbonne 1828 formulierte: "Die Idee des Fortschritts, der Weiterentwicklung scheint mir die in dem Wort Zivilisation 
enthaltene grundlegende Idee zu sein «; sie umfasst zwei Dimensionen: die Entwicklung der menschlichen Gesellschaft und die des Menschen selbst. Alles in allem »ist es die Idee eines Volkes, das voranschreitet, aber nicht, um den Platz zu wechseln, sondern seinen Zustand«. Sodass »eine Universalgeschichte der Zivilisation zu schreiben« wäre. ${ }^{15}$ Der Philosoph Marcel Gauchet hat diesen sich Mitte des 19. Jahrhunderts eröffnenden Moment als den des Übergangs des "politischen Verhältnisses« zum "historischen Verhältnis« bezeichnet. ${ }^{16}$ Vergessen wir allerdings nicht, dass für Leopold von Ranke, den immer noch berühmten Vater der modernen Geschichtsforschung, die Hegelsche Sicht der Geschichte als Fortschritt des Geistes untragbar war: wäre sie doch sonst auf einen "werdenden Gott" hinausgelaufen. »Ich meinerseits«, fährt er fort, "glaube an den, der da war und da ist und seyn wird, und an die wesentliche unsterbliche Natur des individuellen Menschen. ${ }^{17}$ Dennoch gibt es eine Universalgeschichte (die $\mathrm{zu}$ schreiben er auf seine alten Tage ja selbst begonnen hatte), verstanden schlicht und einfach als Zusammenfügen der Ereignisse aus allen Zeiten und von allen Nationen; dies eine klassische Auffassung, die sich bis mindestens Diodoros von Sizilien zurückverfolgen lässt. Allerdings fügte Ranke ein doppeltes caveat hinzu: sofern man sie wissenschaftlich behandeln kann und vorausgesetzt, man trennt nicht die Erforschung des Einzelnen vom Ganzen, an das es gebunden ist. Damit war den immer wieder aufflammenden Debatten über das Allgemeine und das Besondere reichlich Stoff geliefert.

Die philosophische Weltgeschichte, jene, auf die das moderne Europa sein Leben aufgebaut hat, und zwar in einem Maße, dass es sie zu absolutieren oder zu naturalisieren suchte, um sie zum Maßstab aller Geschichte zu erheben, ist primär gekennzeichnet durch die Rolle, die der Zukunft zugeschrieben wird: Sie ist zukunftszentriert oder zukunftsorientiert, das heißt vom Gesichtspunkt der Zukunft aus entworfen. Zur Sache des Philosophen erklärt, stellt sie vielleicht den treffendsten Ausdruck des ıneuzeitlichen Historizitätsregimes` dar. So setzt etwa Schiller in seiner Jenaer Antrittsvorlesung von 1789 »Was heißt und zu welchem Ende studiert man Universalgeschichte« als evident: „Die Entdeckungen, welche unsre europäischen Seefahrer in fernen Meeren und auf entlegenen Küsten gemacht haben, [...] zeigen uns Völkerschaften, die auf den mannigfaltigsten Stufen der Bildung um uns herum gelagert sind, wie Kinder verschiednen Alters um einen Erwachsenen herumstehen «. ${ }^{18}$ Beschleunigung, Verzögerung, Fortschreiten, später Aufholen werden operative Begriffe ${ }^{19}$ Es obliegt dem "philosophischen Verstand«, all diese Beobachtungen und historischen Bruchstücke, dieses "Aggregat zum System " zu erheben. ${ }^{20}$ Denn der Philosoph allein ist in der Lage, die Geschichte in ihrer Gesamtheit zu erfassen. Gedacht als »Auslegung des Geistes in der Zeit«, verläuft die Weltgeschichte bekanntlich »von Osten nach Westen, denn Europa ist schlechthin das Ende der Weltgeschichte, Asien der Anfang «. ${ }^{21}$ Man dürfte Hegel wiedererkannt haben. Einige sind ihm vorhergegangen, viele andere sind ihm gefolgt, viele haben ihn kritisiert, aber er ist und bleibt in dieser Angelegenheit der Hauptbezugspunkt: derjenige, der bereits vorliegende Vorschläge am weitesten vorantreibt, derjenige, den es zu widerlegen gilt, derjenige, der vom Kopf auf die Füße gestellt werden muss. 


\section{In Verruf geraten}

16 Selbstverständlichkeit dieser Geschichtsphilosophien vorbei; von der Realgeschichte widerlegt, bekommen sie Risse und lösen sich am Ende auf, auch wenn in Deutschland Theologen und Historiker das Problem nicht auf sich beruhen lassen. ${ }^{22}$ Der Erste Weltkrieg hat all diese Gebäude in ihren Grundfesten erschüttert, so wie die Statue mit den tönernen Füßen in Nebukadnezars Traum aus dem Buch Daniel zermalmt wird. Einer der ersten, auf den wir in diesen Gefilden stoßen, ist Oswald Spengler, dessen Untergang des Abendlandes den Untertitel trägt: Umrisse einer Morphologie der Weltgeschichte. Theodor Lessing wiederum stellt die Geschichtswissenschaft ganz in Frage, indem er aufzeigt, dass sie nicht in den Bereich der Wissenschaft, sondern in den des Glaubens gehört: Geschichte als Sinngebung des Sinnlosen heißt sein während des Krieges verfasstes und 1919 erschienenes Werk. Die zunächst 1919 auf Englisch veröffentlichten Überlegungen Valérys über "Die Krise des Geistes« geben die Diagnose eines Zusammenbruchs: „Es bedurfte zweifellos vielen Wissens, um in so kurzer Zeit so viele Menschen zu töten, so viel Güter zu verschwenden, so viele Städte zu vernichten; aber nicht weniger bedurfte es dazu moralischer Kräfte. Wissenschaft und Pflicht, seid auch ihr nun verdächtig? «23

17 Vor dem Krieg verfasst, aber erst 1918 veröffentlicht, ist Spenglers umfangreiche Abhandlung von dem Ehrgeiz getragen, eine vergleichende Geschichtsmorphologie der Kulturen $\mathrm{zu}$ begründen. Dabei greift Spengler auf das Werkzeug der Geschichtswissenschaft, die Analogie, zurück, wie er auch die gesamte Untersuchung auf die nach ihm unbestreitbare Parallele zwischen den Jahrhunderten des Untergangs der Antike und der beginnenden Phase der Weltgeschichte begründet. Die Zeit verwandelt sich dementsprechend in eine "Logik des Schicksals«, und dieser prognostisch ambitionierte neue Blick auf die Geschichte will nichts weniger sein als eine »Philosophie des Schicksals«. Um eine Wendung Raymond Arons aufzugreifen: Ausgehend von einem metaphysischen Dekret über die Wirklichkeit der Kulturen verwirft und widerlegt Spengler den rationalistischen Optimismus des Abendlandes.

Arnold Toynbee, den dieser kulturgeschichtliche Ansatz zunächst begeistert hatte, wird vom Ersten Weltkrieg unmittelbar betroffen. Die Wirklichkeit des Todes trifft mehrere seiner Oxforder Gefährten; darüber hinaus verspürt er so etwas wie den Selbstmord des »Gangs der Freiheit«. »Auch wir waren sterblich" (wir, aber auch unsere Kulturen), wird er im Rückgriff auf Valérys berühmten Anfangssatz aus der »Krise des Geistes« sagen und damit seine Erfahrung der gleichen Ereignisse zum Ausdruck bringen. Von Hause aus Hellenist, geht Toynbee wie Spengler von der Parallele zwischen dem Peloponnesischen Krieg und dem Ersten Weltkrieg aus, bevor er dann den Primat der westlichen Zivilisation in Zweifel zieht und sich dem Gang der Weltgeschichte widmet, einer immensen Studie der vielfältigen Formen von Aufstieg und Niedergang der Zivilisationen. ${ }^{24}$ Legt man die Dauer der Geschichte der Erde und der Menschheit als Maßstab an die verschiedenen Zivilisationen an, kann man durchaus von ihrer "philosophischen Zeitgenossenschaft« sprechen. So steht nichts im Wege, zwischen der Lage im Jahr 1914 und jener in Griechenland zum Zeitpunkt des Peloponnesischen Krieges eine Analogie zu postulieren: Die griechische Vergangenheit konnte sehr wohl unsere Zukunft sein. 
19 Wer zur selben Periode auf feste, direkt mit dem ıneuzeitlichen Historizitätsregimer und dessen Zukunftsverheißungen verbundene Gewissheiten stoßen will, muss den Blick auf den Marxismus-Leninismus richten, auf das Land der proletarischen Revolution, die Sozialistische Internationale und die weltrevolutionären Projekte wie auch auf die diversen Parteien und Gruppierungen, die sich auf vielfältige Formen kommender Revolutionen berufen. Das Verhältnis der Faschismen zur Zeit ist dagegen ein anderes: Hier vermischen sich Futurismus, nostalgisches Festhalten am Vergangenen und Glorifizierung der Gegenwart vermittels der charismatischen Figur des Führers. In einem gewissen Sinne ist das nazistische »Tausendjährige Reich» von Beginn an, schon 1933, da, aber es ist zugleich auch Rückkehr und Zukunft. ${ }^{25}$

Lässt man den Zusammenbruch der faschistischen Regierungssysteme und das Vordringen der Revolution nach 1945 einmal beiseite, formiert sich Kritik gegen die vorgeblichen Evidenzen der Geschichte (die der großen Geschichtsphilosophie des vorhergehenden Jahrhunderts) im Kontext der Nachkriegszeit und der Entkolonisierung. Etwa jene, die mehrfach ein zum Ethnologen gewordener Philosoph, Claude Lévi-Strauss, äußert, zumal in einer kurzen, polemischen Schrift, die er $1952 \mathrm{im}$ Auftrag der UNESCO verfasste: Rasse und Geschichte. ${ }^{26}$ Halten wir fest, dass damals ein Anthropologe und kein Philosoph, aber auch kein Historiker darum gebeten wurde, einen allgemeinen Rahmen für Überlegungen vorzulegen. Von Welt- oder Universalgeschichte ist in jenem Bändchen nicht die Rede (das Wort selbst fällt nie), aber der Leser wird aufgefordert zur Kenntnis zu nehmen, dass "wir» die »erste Weltzivilisation" sind. Die Zivilisationen verteilen sich, so der Autor, weniger in der Zeit als im Raum. Und schon ist der Evolutionismus verabschiedet, genauer: jener falsche Evolutionismus, der uns z. B. glauben machen wollte, dass die Steinzeit bei den Ureinwohnern Australiens wiederzufinden sei. Und der Zivilisationsprozess ist weniger als ein kontinuierlicher und kumulativer Verlauf zu betrachten denn als eine Schachpartie, bei der jede Gesellschaft einmal gewinnt und einmal verliert.

21 Wenn Lévi-Strauss im Verlauf seiner Interventionen auffordert, Fortschritt nicht als universelle Kategorie zu begreifen, sondern als »besonderen Existenzmodus [...], der unserer Gesellschaft [...] eigen ist «, dann macht er nichts anderes als - in meinen Worten - das sneuzeitliche Historizitätsregime` vehement in Zweifel $\mathrm{zu}$ ziehen. ${ }^{27}$ Gleiches gilt für seine (rasch berühmt gewordene) Unterscheidung zwischen warmen und kalten Gesellschaften, deren Tragweite, wie er immer wieder hervorgehoben hat, theoretischer Natur ist. Wurden die einen durch die bereits erwähnte Verzeitlichung der Geschichte tatsächlich gestaltet, wurde diese von ihnen sogar zu einem Entwicklungsprinzip erhoben, so ist dies bei den anderen Gesellschaften nicht oder noch nicht oder nur mit Schwierigkeiten und partiell eingetreten; dennoch steht fest, dass alle gleichermaßen in der Geschichte stehende und Geschichte stiftende Gesellschaften sind, jedoch mit unterschiedlichen Seinsweisen im Verhältnis zur Zeit. ${ }^{28}$ Dieses Modell, das durchaus nicht dichotomisch verfährt, hat den Vorzug, dass es die Formen und Modalitäten zu verstehen hilft, wie die verschiedenen Gesellschaften zur Zeit stehen: Es hätte zu Vergleichen anregen können. Auch zeigt sich hier - in Gegensatz zu dem, was am Strukturalismus zunächst bemerkt wurde (sein Antihistorismus) - ein wahres Interesse an der Zeit und an der Unterschiedlichkeit von Zeitrhythmen.

22 Zwischen der Haltung von Lévi-Strauss und der von Braudel ist eine Konvergenz feststellbar: eine grundsätzliche Kritik am ıneuzeitlichen Historizitätsregimer. 
Während Braudel nun aber in der Zeit Europas bleibt (auch wenn er sie streckt und anpasst, indem er seine Figur des Mittelmeers, dann den Begriff der Welt-ökonomie entwickelt), bevor er am Ende seiner Reisen zum Problem der Nationalgeschichte eben gerade im Licht der longue durée zurückkehrt, macht Lévi-Strauss gewissermaßen eine Kehre: vom Proletarier zum Wilden oder von Marx zu Rousseau. Die volle Anerkennung des Menschseins des Wilden ist eine bestimmte Art und Weise, den Humanismus, der sich als unzulänglich erwiesen hat, durch seine Erweiterung $\mathrm{zu}$ erneuern (zu entsühnen). ${ }^{29}$

Bei den Philosophen ist nun nicht mehr die Rede davon, zu verkünden oder auch nur anzunehmen, die Geschichtsphilosophie oder die Universal- bzw. Weltgeschichte sei ihre ureigenste Sache. ${ }^{30}$ Wenn es denn überhaupt Hoffnung gibt, dann liegt sie in den Händen der Arbeiterklasse. Sartre mühte sich damit ab, eine Philosophie der Geschichte zu erarbeiten, die Leser Heideggers begaben sich gar nicht erst auf dieses Terrain. 1975 erinnerte Michel Foucault daran, dass Edmund Husserl bereits Mitte der 30er Jahre des 20. Jahrhunderts »das gesamte Wissenssystem, dessen Zentrum Europa gewesen und durch das es sowohl befreit als auch eingesperrt war", in Frage gestellt hatte. Die Geschichtsphilosophien waren zugleich Träger der Befreiung und der Gefangennahme gewesen, und das nicht nur in einem metaphorischen Sinn. Foucault fuhr fort:

»Für uns tauchte dieses Fragen wenige Jahre nach dem Krieg und nach allem, was geschehen war, in seiner ganzen Lebendigkeit wieder auf. Einbruch einer ganz und gar zeitgenössischen Geschichte in eine höchst akademische Philosophie: Was waren dieses Wissen und diese Rationalität, die so tiefgründig mit unserem Schicksal verknüpft und so ohnmächtig angesichts der großen Geschichte waren? Und natürlich waren die Humanwissenschaften solche Objekte, die durch dieses Vorgehen sich in Frage gestellt sahen. "31 $^{31}$

Zwar skizzierte er damit sein eigenes Arbeitsprogramm, doch seine Diagnose galt durchaus nicht nur für ihn. Was die Historiker anbetrifft, so ziehen ein Braudel, ein Chaunu sie aufs offene Meer hinaus, es geht darum, die Geschichte der europäischen Expansion auf andere Weise wieder aufzunehmen, aber im eigentlichen Sinn nicht darum, eine Universalgeschichte zu denken.

\section{Eine Wiederaufnahme?}

Überspringen wir einmal die Jahrzehnte des siegreichen Strukturalismus, die die Infragestellung des Subjekts, aber auch, nach einem letzten Aufflammen, das Erlöschen der Revolutionsidee erleben, dann ist der Beobachter frappiert vom plötzlichen Wiederauftreten der Geschichtsphilosophie kurz nach 1989 (mit dem Fall der Berliner Mauer als Symbol). Diesmal geht die Initiative von Politologen aus. Mit dem Artikel von Francis Fukuyama »The End of History?« in der Rolle des Herolds, der vermutlich in weniger als 80 Tagen die Reise um die Welt machte. ${ }^{32}$ Immerhin lässt diese Rezeption eines missverstandenen Titels sich bei aller verworrenen Überstürztheit als Indiz verstehen. Von Interesse für uns ist die allgemeine These insofern, als sie eine Verteidigung der Existenz einer Universalgeschichte darstellte und deren Wiederaufnahme sein wollte. Tatsächlich war es die Absicht des Autors, an aufgegebene, wenn nicht gar schon lange in Misskredit geratene Überlegungen wieder anzuknüpfen und auf seine Weise den weltbürgerlichen Standpunkt Kants (in dessen kleiner Schrift von 1784) aufzugreifen. ${ }^{33}$ Die Geschichte, ein einfacher und in sich 
stimmiger Prozess, führt, indem sie die Erfahrung aller Völker zur gleichen Zeit berücksichtigt, zwangsläufig den größten Teil der Menschheit zur liberalen Demokratie. Die Geschichte ist gewiss nicht abgeschlossen, beileibe nicht, aber man weiß fortan, dass es zur liberalen Demokratie als deren telos keine Alternative mehr gibt. Mit gutem Grund darf man also in der Geschichte einen kohärenten Prozess am Werk sehen.

Doch lediglich vier Jahre nach dem Buch Fukuyamas findet eine weitere Formel ein weltweit vermutlich noch breiteres und vor allem dauerhafteres Echo, können sich dieses Modell doch noch mehr Protagonisten zu eigen machen und für sich beanspruchen. Auch dieses Phänomen stellt ein Indiz dar. Ebenfalls aus den Vereinigten Staaten stammend, wird diese These (die wie im vorherigen Fall zunächst in Artikelform kursierte) von einem weiteren bekannten Politologen in Umlauf gesetzt, und zwar von Samuel Huntington, dessen Student Fukuyama übrigens gewesen ist. Es geht um den »clash» (»Kampf«) der Kulturen (und »die Neugestaltung der Weltpolitik im 21. Jahrhundert ", wie es im Untertitel heißt). ${ }^{34}$ Jetzt befindet man sich nicht mehr auf der Seite von Kant und der Aufklärung, nicht einmal mehr auf der Hegels, sondern in gewisser Hinsicht in einer deutlich späteren Epoche, nämlich der Spenglers und Toynbees. Nicht ganz zu Unrecht hat denn auch der französische Politologe Pierre Hassner den Autor als "Spengler für die Zeit nach dem Kalten Krieg « qualifiziert. ${ }^{35}$ Denn Huntington, der den kulturspezifischen Ansatz wiederaufgreift und in Kraft setzt, bringt einen holistischen Ansatz ins Spiel, um diese Welt am Ende des 20. und Anfang des 21. Jahrhunderts zu erfassen, in der "politische Orientierungen, die durch Ideologie [...] definiert waren, [...] Orientierungen Platz (machen), die durch Kultur und Zivilisation definiert werden «. ${ }^{36}$ Überzeugt davon, dass selbst vereinfachte "Paradigmen« oder "Karten« für den unabdingbar sind, der die Welt verstehen und $a$ fortiori für den, der auf sie einwirken will, zitiert der Politologe zustimmend Braudel, der hervorhebt, dass man zunächst auf einer Weltkarte die Kulturen - jene umgreifenden Realitäten von langer Dauer - ausmachen muss, die es heutzutage gibt. ${ }^{37}$ Dank des kulturellen Paradigmas kann man zum Beispiel festlegen, wo Europa endet (dort, wo die okzidentale Christenheit aufhört und die Orthodoxie und der Islam beginnen). Aus einer solchen Herangehensweise ergibt sich, entsprechend einer alles in allem wenig originellen Definition, dass die Kulturen die umfassendsten "Menschenstämme« bilden und dass der Zusammenprall und Kampf der Kulturen nichts anderes ist als ein "Stammeskonflikt« auf globaler Ebene. Eine solche auf Zivilisationen basierende internationale Ordnung erzeugt Instabilität, ist zugleich aber auch »der sicherste Schutz vor einem Weltkrieg «. ${ }^{38}$ Da die Zeit der Eroberungen zu Ende sei, plädiert Huntington für einen Stellungskrieg, wobei er die Theorie des containment auf der Ebene der Kulturen recycelt.

Was also tun? Sich bewusst werden, dass die Welt dabei ist, moderner und weniger westlich zu werden, und dass es zwar Kulturen gibt, sich die Vorstellung aber, dass die Welt ein und dieselbe universale Kultur sei, nicht mehr verteidigen lässt. Daher die Botschaft an die Adresse der Amerikaner: "Das Überleben des Westens hängt davon ab, daß die Amerikaner ihre westliche Identität bekräftigen und die Westler sich damit abfinden, daß ihre Kultur einzigartig, aber nicht universal ist, und sich einigen, um diese Kultur $\mathrm{zu}$ erneuern und vor der Herausforderung durch nichtwestliche Gesellschaften $\mathrm{zu}$ schützen. ${ }^{39}$ Was noch? Faktisch hat Huntington einen »inneren Feind« im Visier, nämlich die Verfechter des Multikulturalismus, die in der westlichen 
Erbschaft lediglich die Verbrechen des Westens sehen. Sie wollen »die Amerikaner von ihrem sündigen europäisches Erbe [...] befreien und erlösende Infusionen aus nichtwestlichen Kulturen anbringen ${ }^{40}{ }^{40} \mathrm{Er}$ zitiert Arthur Schlesinger und dessen Verweis auf die Devise der Gründungsväter »E pluribus unum«. Zerfiele Amerika jemals in eine Vielzahl von Kulturen, wäre es nicht mehr die Vereinigten Staaten, sondern die Vereinten Nationen. Was deutlich macht, dass Huntingstons Hauptziel in dem Aufruf besteht, die westliche Kultur zu bewahren, zu schützen, zu stärken - und zwar von den Vereinigten Staaten aus. ${ }^{41}$ Diese Haltung, die eine gewisse Furcht vor der Zukunft durchblicken lässt, ist zugleich auch Aufforderung zu einem Rückzug. Der zentrale Unterschied zu Fukuyama ergibt sich aus ihrem Spenglerschen Ansatz. Sind die Kulturen tatsächlich jene umfangreichsten »Menschenstämme«, und lässt sich der Zusammenstoß, der Kampf der Kulturen als "Stammeskonflikt auf globaler Ebene« begreifen, dann ist der Verzicht auf den Universalismus der Preis, den der Westen zu zahlen hat, um sich besser zu verteidigen, das heißt, um besser ein Amerika zu schützen, das der tödlichen Falle des Multikulturalismus entgehen könnte, indem es nachdrücklich seine Zugehörigkeit zur westlichen Kultur geltend macht. Die westliche Kultur ist einzigartig, aber nicht universal. ${ }^{42}$ Das Vorhandensein von Kulturen widerspricht universalistischen Ansprüchen, und somit gibt es weder eine Universalkultur (ein falscher und gefährlicher Begriff) noch eine Universalgeschichte. In ihrer robusten Schlichtheit mag diese These im Norden wie im Süden vielen gelegen kommen.

\section{»Global History«}

Diese Jahre sind zugleich jene, in denen die "Globalisierung" im Mittelpunkt des öffentlichen, aber auch akademischen Interesses steht, mit dem "www« als Erkennungszeichen und machtvollem Instrument. Haben wir es mit einem Kofferbegriff zu tun, in den jeder hineinlegt und herausnimmt, was er will? Sicher. Ist es eine bestimmte Art und Weise, zunächst einmal die Welt von den Vereinigten Staaten aus zu begreifen (wie auch der Aufschwung der Empire-Thematik zu belegen scheint)? Ja, aber nicht nur. Der Aufstieg vor allem Chinas, Indiens, Brasiliens hat »die lokalen und nichtwestlichen Formen in den Dynamiken der Globalisierung « sichtbar werden lassen. ${ }^{43}$ Eine Vielzahl von Arbeiten widmet sich seit zehn bis fünfzehn Jahren diesem Thema, und ihre Zahl steigt rasant.

Beschränken wir uns auf die professionelle Geschichtswissenschaft: Hier ist eine Geschichte auf die Bühne getreten, die sich als "globale« bezeichnet, während sich zugleich eine »World History« erneut regte oder doch einen neuen Anlauf nahm. Die eine wie die andere mit ihren jeweiligen Zeitschriften, Publikationen, Vereinigungen, Homepages, ihren Zentren, Studiengängen, Kongressen, ihren (neuen oder alten) Experten, wie auch Anfängen einer Selbstreflexion. Patrick Manning hat eine erste gleichermaßen retrospektive wie prospektive Kartographie der globalen Geschichte vorgelegt. Sein 2003 veröffentlichtes Buch trägt den sprechenden Titel: Navigating World History. Der world historian oder der, der es werden will, kann damit lernen, dieses Softwareprogramm »world« oder »global« History zu meistern, besser zu benutzen, ja zu entwickeln. Der Untertitel versetzt uns dann unmissverständlich in eine konstruktivistische Perspektive: Historians Create a Global Past. ${ }^{44}$ Um sich dieser weiträumigen Verschiebungen zu vergewissern, hat der Geschichtshistoriker Georg 
Iggers in seinem steten Bemühen, dem Kommenden einen Platz einzuräumen, $z u$ verstehen und anderen verständlich zu machen, ohne doch je auf die Geschichte und auf seine Verantwortung als Historiker zu verzichten, zusammen mit zwei Kollegen eine Global History of Modern Historiography veröffentlicht. ${ }^{45}$ Iggers, der es zu Beginn seiner Laufbahn unternahm, den Amerikanern den Historismus zu erklären, sieht seine heutige Aufgabe darin, einen globalen Ansatz der Historiographie vorzulegen. Womit wir bei den Antipoden der Voraussetzungen des Historismus angelangt wären.

Was also ist ein Gobalhistoriker? Ein Historiker der Globalisierung, könnte man antworten. Faktisch nimmt die Globalisierung heute einen der Modernisierung der fünfziger Jahre (ja der Zivilisation bzw. Kultur Anfang des 19. Jahrhunderts) entsprechenden Platz ein: Ein und dasselbe Suffix zeigt in beiden Fällen an, dass der Prozess selbst Gegenstand der Untersuchung ist. Eine gewisse Analogie ließe sich auch in der jeweiligen Einstellung $\mathrm{zu}$ den beiden Phänomen ausmachen: von bedingungsloser Zustimmung über verschiedene Versuche, sie im Plural zu denken, bis hin zu völliger Ablehnung. Wie es nicht nur eine Modernisierung und nicht nur eine einzige Moderne gab, so gibt es auch keine Globalisierung, die zur gleichen Zeit die gesamte Welt auf einmal gewissermaßen überrennen würde. Eine ganze Reihe von Analysen sind entwickelt worden, die auf das binäre System des Globalen und des Lokalen setzen: Effekte des Globalen auf das Lokale, das Glokale, und ein gewisser Rückkoppelungseffekt dieses Glokalen auf das Globale.

Nach Bruce Mazlich, einem der ersten Vertreter und Fürsprecher der Globalgeschichte, weist das Adjektiv "global« in Richtung eines Raums (des Globus). Globalgeschichte zu praktizieren heißt denn auch, ihm zufolge, das »Raumschiff Erde" gleichsam von einem Satelliten aus zu beobachten; dies ist für die Untersuchung einer bestimmten Zahl von Prozessen auch die sachgerechteste Perspektive. Sehr schön, aber wie, so wird man fragen dürfen, verwandelt sich der Historiker in einen Astronauten oder in einen Satelliten? Wie konstruiert er diesen Blickpunkt »im Weltraum«, dabei überzeugt, dass er nicht nur (in neuer Aufmachung) schlicht den abgehobenen Blickpunkt einnimmt, den sich der Historiker unlängst noch zuschrieb: ein Surrogat des göttlichen Blicks von außen, des Blicks der antiken Fortuna für Polybios oder des modernen Geistes für Hegel? Trotz alldem ist die Globalgeschichte, so behaupten ihre Wortführer, nicht whiggish oder besser: "welche Gestalt sie annehmen wird, kann nicht vorausgesagt werden«: Der Globalhistoriker weiß »der Kontingenz und der Unsicherheit der menschlichen Angelegenheit« ihren Platz einzuräumen. Er glaubt weder an die Parallele noch an die Analogie. Ein zweites Merkmal, noch immer Mazlich zufolge: Die World History beginnt in der Gegenwart, identifiziert als Eröffnung - um 1970 herum einer "globalen Epoche«, als hinreichend "Synergie und Synchronie« bestehen. Daher "müssen wir«, auch wenn Welt- und Globalgeschichte "sich in einem Kontinuum entfalten, uns bewusst sein, dass wir eine tatsächliche Grenze überschreiten, wenn wir in die Geschichte der Globalisierung oder in die Globalgeschichte eintreten «. ${ }^{46}$

31 Ein 2002 von A. G. Hopkins herausgegebenes Sammelwerk, Globalization in World History, wiederum legt die Betonung auf das Kontinuum, wobei ausgehend vom Ansatz der longue durée die »Illusion« kritisch hinterfragt wird, »die in der Globalisierung lediglich ein Geschöpf des Okzidents sehen wollte«. Die Autoren machen so mehrere Globalisierungsschübe aus: eine archaische, eine Proto-Globalisierung (zwischen 1600 und 1800), eine neuzeitliche (die der Nation und der Industrialisierung entspricht), eine post-koloniale (in den 50er Jahren) und eine neue Phase nach $1970^{47}$ - jene, die, um 
überhaupt erfasst werden zu können, für Mazlich einen Satelliten erforderlich macht! Eine multiple Globalisierung zu behaupten heißt, sie zu banalisieren (es gab immer schon Globalisierung), sie zu periodisieren aber könnte dazu führen, sich zu fragen, welche Temporalitäten während jeder dieser Phasen wirksam waren. Um auf meinen Leitgedanken zurückzukommen: Unter welches $\gg$ Historizitätsregime lassen sie sich jeweils fassen?

Wenn die moderne und die post-koloniale Globalisierung vollkommen zukunftsorientiert waren, Zukunft als Führungs- und Produktionsinstanz von Intelligibilität einführten, wie steht es dann um die aktuelle Phase, also die seit 1970? Dass jemand im Westen darauf verfallen könnte, an die Geschichtsphilosophien des 19. Jahrhunderts und die Kolonialreiche gebundene, in den Universalgeschichten eingesetzte Zeitschemata erneut in Umlauf zu bringen, ist ja ausgeschlossen. Dies umso mehr, als Europa in jenen Jahren die Erfahrung einer Abschließung des Zukunftshorizonts und eines Relevanzverlusts der Kategorie Zukunft machte. Bewegt die aktuelle Phase sich im Präsens, in diesem omnipräsenten zeitgenössischen Präsens? Ist sie, um nochmals auf meine Wortwahl zurückzugreifen, präsentisch? Ist das Präsens der Modus, in dem sie sich fassen lässt?

Dieser Situation, in der Globalisierung und Fragmentierung Hand in Hand gehen, suchte die Lösung bzw. Versuchung der Postmoderne zu begegnen. Ihr zufolge sollte es letztlich keine Geschichte mehr geben, sondern nur noch Umgangsweisen mit der Vergangenheit. Vielzählig, vielfältig, vielstimmig, wie sie nun einmal sind, bilden sie sich als Erinnerung und im Namen der Identität heraus: jedem seine eigene Erinnerung. Eingesetzt im Präsens und in dessen Horizont: präsentisch von Grund auf. Unter Berufung auf die Erinnerung werden nicht nur alternative Geschichten gefördert (zumal im Gefolge der wichtigen Bewegung der subaltern studies), sondern schlichtweg Alternativen zur Geschichte. Die Erinnerung wider die Geschichte, die stets noch die der Sieger ist. Ein Ansatz, den der indische Intellektuelle Ashy Nandy vertritt, für den das historische Bewusstsein überall in der Welt den Sieg davongetragen hat, selbst in Ländern wie Indien, die sich lange Zeit außerhalb der Geschichte gehalten hatten. ${ }^{48}$ Kurioserweise findet eine solche kritische Haltung am Ende wieder Anknüpfungspunkte zu Hegel, für den die Geschichte schlechthin doch gerade ein Erbteil Europas war! Zwar verläuft der Anschluss an das historische Universum über ein und denselben Weg, aber jede Kultur, so Nandys These, verfügt über eine bestimmte Art und Weise, sich in ihrer A-Historizität einzurichten. ${ }^{49}$ Der auf Gegensätzlichkeit und Komplementarität beruhende Austausch vollzieht sich zwischen Differenzen und Uniformisierung.

Weitaus weniger radikal ist der Rückgriff auf den Begriff der »connected histories« mit dem Ziel, zunächst die zeitgenössische Geschichte zu schreiben, dann aber auch, sich aufs Neue den Geschichten der Vergangenheit zuzuwenden. Mit dieser methodologischen, ja nahezu technischen Proposition sind wir wieder im Kernbereich der Zunft der Historiker. ${ }^{50}$ "Connected«, das heißt »verbunden «, »zusammenhängend«, "verflochten", "vernetzt", ist allerdings kein neutrales Wort, vielmehr ein höchst aktueller Begriff: positiv (man muss vernetzt sein), aber, so wage ich einmal zu behaupten, recht elastisch. Wo beginnt und wo endet eine zusammenhängende, vernetzte Geschichte? Verbindungen, Verflechtungen, Zusammenhänge auszumachen ist zunächst ein Instrument, allzu einsinnig angelegte, asymmetrische, ungleiche Geschichten neu zu betrachten, zu ersetzen und die Zwangsjacke der nationalen und 
kolonialen Geschichten abzustreifen. Daraus ergibt sich ein Erkenntnisgewinn. Der konnexionistische Historiker betrachtet seine Quellen mit anderen Augen, erweitert damit den Begriff des Dokuments, dynamisiert so seinen Fragenkatalog. Doch die Suche nach Zusammenhängen, die abschließend ein Netz bilden, setzt mitnichten eine Weltgeschichte und die Möglichkeit eines monopolartigen Blicks auf diese voraus. Die Geschichte kann auch nicht nur die - und sei es potenzierte - Summe aller dieser Zusammenhänge sein, da es möglich sein muss, die Verbindungen in Abhängigkeit von den Fragen, die der Historiker stellt, auch anders anzuordnen.

Ist unter diesen Umständen überhaupt eine Form von Weltgeschichte denkbar, die nicht zukunftszentriert und teleologisch wäre? Die sich dennoch weder darauf beschränkte, von einer vollständigen und endgültigen Zersplitterung Kenntnis zu nehmen (um dies zu begrüßen oder aber zu bedauern), noch sich in die Verliese der longue durée der Kulturen einschließen ließe. Die sich auch nicht damit begnügte, die alte zeitliche Schichtung der Zivilisationen (vom Primitiven zum Zivilisierten) durch eine bloße räumliche Aufteilung (entsprechend dem Vorschlag von Lévi-Strauss) zu ersetzen. Die nicht voraussetzen dürfte, dass derjenige, der sie schreibt, sich - und sei es unausgesprochen oder zufällig - einen übergreifenden Gesichtspunkt vorgibt. Könnte sie nicht am Ende die Rolle einer regulativen Idee spielen? Der Rückgriff auf den Begriff des >Historizitätsregimes beansprucht selbstredend nicht, die Lösung zu sein, das aus dem Zylinder gezauberte Kaninchen, aber er könnte ermöglichen, mit der Arbeit zu beginnen, sich mit der Frage zu beschäftigen, wo die Formen von Zeitlichkeit auseinanderlaufen und zusammenkommen, hier und dort, also wo die 'Historizitätsregime` interagieren, sich verflechten, überlappen, ineinanderfügen, auseinanderstreben, sich voneinander abkoppeln. Sie könnte ermöglichen - ein alter Traum -, Raum und Zeit zu verbinden. Denn wenn es auch keine einzige Zeit gibt, wenn die Erfahrungen von Zeit hier und dort, gestern und heute so ungemein unterschiedlich waren und sind, so gibt es doch überall homologe Weisen, menschliche oder soziale Zeit in einer Welt zu stiften, die immer schon auf einem mehr oder minder hohen Niveau Austausch, Interaktionen, Konflikte gekannt hat.

\section{BIBLIOGRAPHIE}

Assayag, J. (2005): La Mondialisation vue d'ailleurs. L'Inde désorientée, Paris: Seuil.

Braudel, F. (1990 [1949]): Das Mittelmeer und die mediterrane Welt in der Epoche Philipps II., übers. v. G. Osterwald u. G. Seib, Frankfurt/M.: Suhrkamp.

Braudel, F. (1992 [1969]): Schriften zur Geschichte 1, übers. von G. Kurz u. S. Summerer, Stuttgart: Klett-Cotta.

Braudel, F. (2009 [1986]): Frankreich, 3 Bde., übers. von P. Schöttler, S. Summerer u. G. Kurz, Stuttgart: Klett-Cotta.

Conrad, S. / Eckert, A. / Freitag, U. (Hg.) (2007): Globalgeschichte: Theorien, Ansätze, Themen, Frankfurt/M.: Campus. 
Foucault, M. (2004 [1975]): Gespräch mit Roger-Pol Droit, Le Point, 1. Juli.

Fukuyama, F. (1989): »The End of History?«, The National Interest, 16, S. 3-18.

Fukuyama, F. (1992): Das Ende der Geschichte. Wo stehen wir?, aus dem Amerikanischen v. H. Dierlamm, U. Mihr u. K. Dürr, München: Kindler.

Gauchet, M. (2005): La Condition politique, Paris: Gallimard.

Gruzinski, S. (2001): »Les mondes mêlés de la monarchie catholique et autres `connected histories «", Annales. Histoire, sciences sociales, 1, S. 85-117.

Guizot, F. (1985): Histoire de la civilisation en Europe, Paris: Hachette.

Hartog, F. (2001): Le Cas Fustel de Coulanges, le XIX ${ }^{e}$ siècle et l'histoire, Neuaufl., Paris: Seuil.

Hartog, F. (2003): Régimes d'historicité, Présentisme et expérience du temps, Paris: Seuil.

Hartog, F. (2005): Anciens, Modernes, Sauvages, Paris: Galaade Éditions.

Hassner, P. (1994): »Un Spengler pour l'après-guerre froide«, Commentaire, 18, S. 263-265.

Hegel (1970 [1840]): Werke in zwanzig Bänden, Bd. 12: Vorlesungen über die Philosophie der Geschichte, Frankfurt/M.: Suhrkamp.

Hopkins, A. G. (Hg.) (2002): Globalization in World History, London: Pimlico.

Huntington, S. P. (1996): Der Kampf der Kulturen: Die Neugestaltung der Weltpolitik im 21. Jahrhundert, aus dem Amerikanischen v. H. Fließbach, München / Wien: Europa-Verlag.

Iggers, G. G. / Wang, E. Q. / Mukherjee (2008): Global History of Modern Historiography, Harlow / München: Pearson / Longman.

Jaspers, K. (1949): Vom Ursprung und Ziel der Geschichte, München: Piper.

Kant, I. (1964 [1784]): »Idee zu einer allgemeinen Geschichte in weltbürgerlicher Absicht «, in: ders.: Kleinere Schriften zur Geschichtsphilosophie, Ethik und Politik, Hamburg: Meiner, S. 3-20.

Koselleck, R. (1975): „Geschichte«, in: Brunner, O. / Conze, W. / Koselleck, R. (Hg.): Geschichtliche Grundbegriffe. Historisches Lexikon zur politisch-sozialen Sprache in Deutschland, Stuttgart: Klett-Cotta, 2. Bd., S. 647-717.

Koselleck, R. (1979 [1977]): »NNeuzeit«. Zur Semantik moderner Bewegungsbegriffe«, in: ders. (1979): Vergangene Zukunft. Zur Semantik geschichtlicher Zeiten, Frankfurt/M.: Suhrkamp, S. 300348.

Koselleck, R. (1997): »Vom Sinn und Unsinn der Geschichte«, Merkur, 4, S. 319-334; auch in: ders. (2010): Vom Sinn und Unsinn der Geschichte. Aufsätze und Vorträge aus vier Jahrzehnten, hg. v. C. Dutt, Berlin: Suhrkamp, S. 9-31.

Kroll, F.-L. (1998): Utopie als Ideologie: Geschichtsdenken und politisches Handeln im Dritten Reich, Paderborn et al.: Schöningh.

Lévi-Strauss, C. (1967 [1958]): Strukturale Anthropologie, aus dem Französischen v. H. Naumann, Frankfurt/M.: Suhrkamp.

Lévi-Strauss, C. (1972 [1952]): Rasse und Geschichte, aus dem Französischen v. T. König, Frankfurt/ M.: Suhrkamp; auch in: ders. (1975 [1973]): Strukturale Anthropologie II, S. 363-407.

Lévi-Strauss, C. (1975 [1973]): Strukturale Anthropologie II, aus dem Französischen v. E. Moldenhauer, H. H. Ritter u. T. König, Frankfurt/M.: Suhrkamp.

Manning, P. (2003): Navigating World History. Historians Create a Global Past, New York: Palgrave. 
Mazlich, B. (1998): „Comparing global history to world history «, Journal of Interdisciplinary History, XXVIII, 3, S. 385-395.

Nandy, A. (1998): »History's forgotten doubles«, in: Pomper, P. / Elphick, R. H. / Vann, R. T. (Hg.) (1998): World History, Ideologies, Structures and Identities, Oxford: Blackwell, S. 160-178.

Ranke, L. v. (1954): »Einleitung zu einer Vorlesung über Universalhistorie«, in: Kessel, E.: »Rankes Idee der Universalhistorie«, Historische Zeitschrift, 178, S. 269-308.

Rüsen, J. (2001): Zerbrechende Zeit. Über den Sinn der Geschichte, Köln: Böhlau.

Rüsen, J. (2006): Kultur macht Sinn. Orientierungen zwischen Gestern und Morgen, Köln: Böhlau.

Schiller, F. (1966 [1789]): »Was heißt und zu welchem Ende studiert man Universalgeschichte«, in: ders.: Werke in drei Bänden, Bd. II, München: Carl Hanser, S. 9-22.

Simiand, F. (1960 [1903]): »Méthode historique et science sociale«, Annales, ESC, 1, S. 83-119.

Spengler, O. (2007 [1918/1922]): Der Untergang des Abendlandes. Umrisse einer Morphologie der Weltgeschichte, Wiesbaden: Matrix Verlag.

Subrahmanyam, S. (1997): »Connected histories: Notes towards a reconfiguration of early modern Eurasia«, in: Lieberman, V. (Hg.) (1997): Beyond Binary Histories. Re-imagining Eurasie to c. 1830, Ann Arbor: University of Michigan Press, S. 289-315.

Subrahmanyam, S. (2005): »On world historians in the sixteenth century«, Representations, 91 (1), S. 26-57.

Toynbee, A. J. (1979 [1934-1954]): Der Gang der Weltgeschichte, übers. v. J. von Kempski, Zürich: Europa-Verlag.

Valéry, P. (1995 [1919]): »Die Krise des Geistes«, in: ders.: Werke, Bd. 7: Zur Zeitgeschichte und Politik, hg. v. J. Schmidt-Radefeldt, Frankfurt/M. / Leipzig: Insel, S. 26-54.

\section{NOTES}

1. Im Original deutsch. [A.d.Ü.]

2. Koselleck (1997).

3. Rüsen $(2006,2001)$.

4. Hartog (2003).

5. Hartog (2003), S. 33-51.

6. Hartog (2003), S. 69-75, mit weiteren Hinweisen.

7. Koselleck( 1975), S.647-717.

8. Koselleck (1979 [1990]), S.349-375.

9. Simiand (1960 [1903]).

10. Braudel (1990 [1949]).

11. Braudel (2009 [1986]).

12. Braudel (1992 [1969]), S. 81 [Übersetzung modifiziert, A.d.Ü.]

13. Hartog (2001).

14. Braudel (2009 [1986]), Bd. 3, S. 480.

15. Guizot (1985), S. 62, 58.

16. Gauchet (2005), S. 9. Vom historischen Verhältnis unterscheidet er das politische Verhältnis, "unser ständiges Verhältnis, jenes, das uns an unsere Vorgänger bindet und durch das wir weiterhin zur selben Menschheit gehören, jenes, das ungeachtet des Umfangs des Wandels bleibt und unsere fundamentale Identität als Akteure des Gemeinsam-Seins definiert«. 
17. Ranke (1954), S. 307.

18. Schiller (1966 [1789]), S. 13.

19. Koselleck (1979 [1977]), S. 325.

20. Schiller (1966 [1789]), S. 20.

21. Hegel (1970 [1840]), S. 96 f. und S. 134.

22. In Deutschland hat der die idealistische Geschichtsphilosophie einer Kritik unterziehende Historismus nicht aufgehört, die Grenzen einer Wissenschaft der Geschichte auszuloten: In welcher Hinsicht und wie weit kann sie Wissenschaft sein? In Frankreich hat die methodische Geschichtswissenschaft jede Art von Geschichtsphilosophie verbannt (auch wenn sie noch immer von Comte geprägt ist).

23. Valéry (1995 [1919]), S. 27.

24. Spengler (2007 [1918/1922]); Toynbee (1979 [1934-1954]).

25. Kroll (1998), S. 32-43.

26. Lévi-Strauss (1972 [1952]).

27. Lévi-Strauss, C. (1967 [1958]), S. 362.

28. Lévi-Strauss, C. (1975 [1973]), S. 39 f.

29. Hartog (2005), S. $16 \mathrm{f}$.

30. Außer vielleicht für einen Philosophen wie Karl Jaspers (1949).

31. Foucault (2004 [1975]).

32. Fukuyama (1989), (1992).

33. Kant (1964 [1784]).

34. Huntington (1996).

35. Hassner (1994), S.263.

36. Huntington (1996), S.193.

37. Huntington (1996), S. 48

38. Huntington (1996), S.12.

39. Huntington (1996), S.19 f.

40. Huntington (1996), S.502.

41. Huntington (1996), S. 513.

42. Huntington (1996), S. $19 \mathrm{ff}$.

43. Assayag (2005), S. 278.

44. Manning (2003); siehe auch für deutsche Leser Conrad /Eckert /Freitag (2007), eine Auswahl von Texten, die alle (außer einem) zunächst auf Englisch erschienen sind.

45. Iggers / Wang / Mukherjee (2008).

46. Mazlich (1998), S. 385-395.

47. Hopkins (Hg.) (2002). Für eine stimulierende Reflexion über die Weltgeschichten der Vergangenheit bzw. die Vergangenheit dieser Geschichten im 16. Jahrhundert, vgl. Subrahmanyam (2005), S. 26-57.

48. Nandy (1998).

49. Nandy (1998), S. 161.

50. Zu diesem Begriff der »connected histories«, vgl. unter anderem Subrahmanyam (1997) und Gruzinski (2001). 
INDEX

Schlüsselwörter : `Historizitätsregime`, Geschichtsphilosophien, Universalgeschichte, Globalgeschichte, Globalisierung

Mots-clés : régime d'historicité, philosophies de l'histoire, histoire universelle, histoire globale, globalisation

\section{AUTEURS}

\section{FRANÇOIS HARTOG}

François Hartog ist Inhaber des Lehrstuhls für antike und moderne Geschichtsschreibung an der Ecole des hautes études en sciences sociales (EHESS). Nähere Informationen finden Sie hier. 